\title{
Mulheres em situação de rua: uma perspectiva de cuidado singularizado, territorial e intersetorial
}

DOI: : $\underline{\text { https://doi.org/10.5935/1984-9044.20200014 }}$

\section{Caroline Ferreira Jorge $e^{1}$ \& Éllen Cristina Riccí - Universidade Estadual de Campinas (UNICAMP)}

Resumo: As políticas públicas direcionadas à população em situação de rua datam de 2008, no entanto o exercício de direitos dessas pessoas é marcado por barreiras preconceituosas e estigmatizadoras. Mulheres em situação de rua, por exemplo, têm em suas histórias de vida episódios de violência e negligência como causas para sua chegada e permanência nas ruas; vivências essas reeditadas na experiência do adoecimento, gestação e cuidado nesse contexto. O presente artigo, resultante de um trabalho apresentado ao Programa de Residência Multiprofissional em Saúde Mental da Unicamp, se propõe a refletir sobre o cuidado da equipe do Consultório na Rua com mulheres em situação de rua, a partir do recorte específico da gestação, do relato de caso e da experiência da autora como residente, terapeuta ocupacional e participante. Como contribuição, ressaltam-se as práticas de cuidado territoriais, integrais e equânimes, que têm como objetivo a cidadania e os modos de produção de vida.

PALAVRAS-CHAVE: saúde mental, pessoas em situação de rua, mulheres, maternidade, terapia ocupacional, residência

\section{Homeless women: a perspective of singular, territorial and intersectoral care}

Abstract: Public policies aimed at the homeless population date back to 2008 , however the exercise of these people's rights is marked by prejudiced and stigmatizing barriers. Homeless women, for example, have episodes of violence and neglect in their life stories as a reason for their arrival and staying on the streets; experiences that are reissued in the experience of illness, pregnancy and care in this context. The present article, resulting from a work presented to the Multiprofessional Residency Program in Mental Health at Unicamp, proposes to reflect on the care of the Street Clinic team with pregnant homeless women, from the report of case and the author's experience as a resident, occupational therapist and participant. As a contribution, we highlight the territorial, integral and equitable care practices, which aim at citizenship and the ways of producing life.

${ }^{1}$ ORCID: https://orcid.org/0000-0002-3057-3649

2 ORCID: http://orcid.org/0000-0003-3471-1479 


\section{Introdução}

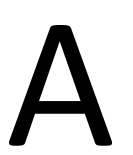

rua, os carros, as placas de sinalização, os prédios, as lojas, as pessoas transitando, conversando, dormindo em bancos ou calçadas; muito ruído de carro e de gente. Essa poderia ser a descrição dos centros das grandes cidades, um cenário comum onde pessoas coexistem, habitam e se relacionam, cada qual com sua marca e história. Pessoas com poder aquisitivo diferentes e, portanto, com diferentes possibilidades de troca. É isso, a rua escancara a diversidade nos modos de produção de vida e de coletivo, assim como as múltiplas funções sociais dos indivíduos na estrutura econômica, política e cultural.

As pessoas em situação de rua, por sua vez, denunciam essa diversidade, a diferença, a existência de padrões de violência, exclusão e negligência. Segundo Surjus e Silva (2019) as comunidades mais marginalizadas em diferentes lugares do mundo possuem em comum um conjunto de fatores, como violência, uso problemático de SPA, barreiras de acesso à cultura e ao trabalho, precarização da saúde, da educação e do transporte público e um alto índice de morbimortalidade que, muitas vezes, é naturalizado por suas populações. Sendo a violência e o uso de SPA principais alvos das ações políticas e comunitárias e não o conjunto de fatores (Surjus e Silva, 2019).

$\mathrm{Na}$ essência, as comunidades marginalizadas e, em especial, as pessoas em situação de rua revelam padrões que, de acordo com Macerata, Soares e Ramos (2014), o bom senso e os costumes não suportam. Mas, se evidenciam algo evitável ou mesmo insuportável, o que garante condições mínimas de visibilidade a essas pessoas? 
MULHERES EM SITUAÇÃO DE RUA: UMA PERSPECTIVA DE CUIDADO SINGULARIZADO, TERRITORIAL E INTERSETORIAL

Como se dá o exercício da cidadania por elas? $\mathrm{O}$ acesso à saúde de fato é um direito?

De acordo com Carvalho (2013), a história da Saúde no Brasil é marcada por ações filantrópicas e intervenções pontuais do Estado diante de epidemias, doenças ou populações específicas, como moradores de rua e prostitutas, aos quais eram destinadas práticas totalizadoras. A compreensão da saúde como direito do cidadão e dever do Estado foi assentada somente quando o SUS, sistema organizado, com diretrizes e princípios delimitados, foi instituído pela Constituição Federal (CF) de 1988 e consolidado pelas Leis 8.080 e 8.142 de 1990 (Carvalho, 2013).

Ainda de acordo com Carvalho (2013), os artigos da CF asseguram o acesso igualitário às ações e serviços de recuperação, proteção, promoção em saúde e atendimento integral. No entanto, os artigos não garantem, em si, o exercício desses direitos, uma vez que pessoas em situação de rua, por exemplo, continuam enfrentando barreiras no acesso a esses serviços por questões de ordem preconceituosa, burocrática e estigmatizadora.

As portarias específicas e políticas públicas direcionadas às pessoas em situação de rua datam de 2008 (Brasil, 2008), mas somente em 2012, com a Política Nacional de Atenção Básica (PNAB) (Brasil, 2012), a responsabilidade sobre a atenção à saúde dessas pessoas foi atribuída a todos os dispositivos de SUS, ou seja, é recente a tentativa de assentar e garantir direitos a essa população. A abrangência das singularidades e necessidades dos sujeitos, nesse contexto, constitui desafio para a saúde coletiva, como, por exemplo, o cuidado da mulher em situação de rua.

Em 2004, foi criada a "Política Nacional de Atenção Integral à Saúde da Mulher: Princípios e Diretrizes", com objetivo de nortear as ações de atenção à mulher, garantir direitos e reduzir a morbimortalidade por causas passíveis de prevenção (Brasil, 2004). Considera, ainda, a diversidade e acúmulo de funções que as mulheres possuem (trabalhadoras, donas de casa, 
MULHERES EM SITUAÇÃO DE RUA: UMA PERSPECTIVA DE CUIDADO SINGULARIZADO, TERRITORIAL E INTERSETORIAL

provedoras dos cuidados), o que ocasiona, muitas vezes, o recorrente acesso aos serviços de saúde e o agravamento de seus processos de adoecimento.

Vale ressaltar que as condições de saúde das mulheres estão sujeitas à discriminação nas relações de trabalho, mercado, lazer, moradia e renda, que, atreladas a um conjunto de variáveis, como raça, etnia e condição social, conformam os indicadores epidemiológicos de adoecimento e morbimortalidade de mulheres. Afinal, “a vulnerabilidade feminina frente a certas doenças e causas de morte está mais relacionada com a situação de discriminação na sociedade do que com fatores biológicos." (Brasil, 2004, p. 9).

As mulheres em situação de rua também compõem tais índices, dado que as situações de discriminação e/ou violência integram suas histórias de vida e, muitas vezes, são a causa de sua chegada às ruas. De acordo com Prudente, Gontijo e Paiva (2018), os meios de chegada de mulheres e homens às ruas são diferentes: os homens vão para as ruas por conta de processos prolongados, problemas familiares, desemprego e uso de Substâncias Psicoativas (SPA), enquanto as mulheres, por rupturas em relação a situações de abuso sexual, violência doméstica e negligência.

Assim, atentando-se aos aspectos citados, pode-se afirmar que cuidar das pessoas em situação de rua, de mulheres nessas condições, é reconhecer que não ocupam somente um lugar físico, mas simbólico, que vincula a rua a outras significações. Isto é, ações exclusivamente assistencialistas não são capazes de refletir ou abranger a complexidade das suas necessidades, como a oportunidade de reinserção nas lógicas das famílias, do trabalho, da moradia, da saúde e das outras esferas de que são excluídas (Brasil, 2011). Ou seja, há necessidade de se pensar como construir uma assistência territorializada, singularizada e articulada com uma rede intersetorial, fundamentada nas diferentes formas de produção de vida.

Quarentei (2001), estudando Guatarri (1992), conceitua a vida como um 
MULHERES EM SITUAÇÃO DE RUA: UMA PERSPECTIVA DE CUIDADO SINGULARIZADO, TERRITORIAL E INTERSETORIAL

continuum incessante de atividades e correlaciona o conceito de Produção de Vida com os diferentes modos de estar e existir no mundo. Compreende, assim, que cada um tem um tempo diferente para se relacionar, interagir e, portanto, vive diversamente os tempos da família, da brincadeira, do desejo e da produção.

Considerando esses aspectos, o presente artigo, derivado do trabalho de conclusão da Residência

\section{O CnaR}

O CnaR, conforme a PNAB (Brasil, 2012), articulado com os serviços da Rede de Atenção em Saúde (RAS), de Atenção Psicossocial (RAPS) e do Sistema Único de Assistência Social (SUAS), atua de forma itinerante, através de uma Unidade Móvel, e tem como população alvo das práticas as pessoas em situação ou circulação de rua, que em comum possuem a pobreza extrema, a ruptura dos vínculos e a ausência de moradia convencional. Por isso, utilizam os logradouros públicos como moradia, de
Multiprofissional em Saúde Mental da Unicamp, tece registros e reflexões acerca do encontro, observação e estudo de uma residente, terapeuta ocupacional, com o território existencial rua e as mulheres que o habitam, observando, sobretudo, o cuidado de uma mulher gestante nessas circunstâncias. Tal encontro foi mediado pela vivência e imersão no cotidiano de trabalho das equipes do Consultório na Rua (CnaR) do município de Campinas.

forma temporária ou permanente; ou estão abrigadas em unidades de acolhimento.

As ações de assistência, promoção e prevenção em saúde realizadas pela equipe do CnaR são organizadas no território onde essas pessoas vivem e baseadas na estratégia transversal de cuidado da Redução de Danos (RD), regulamentada pela Portaria $n^{\circ}$ 1.028/2005. Filho et al (2011) descrevem a RD como contingente de todas as 
MULHERES EM SITUAÇÃO DE RUA: UMA PERSPECTIVA DE CUIDADO SINGULARIZADO, TERRITORIAL E INTERSETORIAL

ações, sendo esta de baixo limiar de exigência e potencializadora da participação dos usuários nos processos de cuidado e na minimização dos danos causados pelo uso das SPA.

De acordo com Valério (2010), citado por Nery Filho et al (2011), são consideradas ações de baixa exigência as propostas flexíveis que incluem ofertas e

\section{Objetivo}

Com o objetivo de observar e refletir sobre o cuidado da mulher gestante em situação de rua, este trabalho foi produzido por uma equipe de CnaR, a

\section{Métodos}

Este artigo se baseia em um estudo qualitativo, descritivo, realizado para conclusão do curso de residência multiprofissional, no qual se utilizou do diário de campo como instrumento de registro e análise dos encontros entre as alternativas preventivas, assistenciais, de suporte psicossocial e de promoção da saúde, com ambientes acolhedores, sem exigências com relação a horários, frequências, abstinências ou outras. São ações de RD todas as intervenções que buscam a orientação, informação, aconselhamento, assistência e disponibilização de insumos de proteção à saúde e prevenção de doenças.

partir do relato de caso e da experiência como terapeuta ocupacional, residente multiprofissional e participante.

equipes do CnaR com mulheres gestantes em situação de rua, partindo da experiência da autora enquanto participante. A adoção desse instrumento se deu pela possibilidade de 
MULHERES EM SITUAÇÃO DE RUA: UMA PERSPECTIVA DE CUIDADO SINGULARIZADO, TERRITORIAL E INTERSETORIAL

observação, descrição e reflexão da situação vivenciada.

Devido à impossibilidade de transcrever todas as anotações, foram selecionados trechos dos encontros com uma mulher gestante, registrada aqui com o nome fictício de Cássia. Conjuntamente, foi realizado um levantamento bibliográfico, que perpassou todo o estudo e, consequentemente, este artigo. No entanto, devido ao recente interesse acadêmico na população estudada, foram poucos os artigos encontrados com os descritores: mulher, rua $\mathrm{e}$ cuidado.

\section{As mulheres e o território rua}

As trajetórias das mulheres assistidas pelo CnaR de Campinas são marcadas por vivências institucionais, nos dispositivos do SUS, SUAS ou do judiciário, visto que elas só são compreendidas como cidadãs a partir da entrada nessas instituições do adoecimento, da fragilidade ou do ato infracional. No entanto, paradoxalmente, as histórias dessas mulheres possuem marcas das ausências das instituições, as quais deveriam defender seus direitos - os direitos à infância, à saúde, à família e aos desejos.

A literatura deflagra que esse não é um percurso comum só das mulheres em situação de rua em Campinas, pois são marcas resultantes de uma organização social patriarcal, machista e sexista, que oprime, violenta e exclui, como extensão de um modo de produção social, não exclusivo da rua, porém escancarado por esse território. A exemplo, esse estudo tomará as condições de acesso das mulheres em situação de rua aos serviços.

A população assistida pelo CnaR de Campinas, segundo os registros de produção diária da equipe, é predominantemente composta por homens. Contudo, isso não necessariamente é representativo da distribuição por gênero das pessoas em 
MULHERES EM SITUAÇÃO DE RUA: UMA PERSPECTIVA DE CUIDADO SINGULARIZADO, TERRITORIAL E INTERSETORIAL

situação de rua no município, pois pode estar atrelado a uma dificuldade de acesso, de vocalizar e validar as demandas das mulheres. Ainda se observa na prática que, quando as mulheres acessam o serviço, seus cuidados, muitas vezes, se dão por ações direcionadas aos cuidados à saúde sexual, diferentemente das inserções dos homens.

Um exemplo dessa característica se dá na experiência da equipe do CnaR de Campinas na oferta de métodos contraceptivos: há uma obrigatoriedade na periodicidade da oferta de contraceptivos injetáveis às mulheres, assim como há necessidade de uma rotina de trabalho que inclui buscas ativas, atendimentos, entre outros. Já as ofertas de preservativos masculinos se dão por livre demanda. Não é objetivo negar as diferenças entre as necessidades e demandas das mulheres e homens ao apontar tais divergências, no entanto, busca-se problematizar como essas diferenças orientam o trabalho e quais as vias de mobilização dessas práticas (organização social, preconceitos).
Outro aspecto pertinente a essa população está atrelado ao circuito social e aos processos de chegada às ruas. Constata-se que muitas mulheres na rua não identificam uma rede de suporte que não as estabelecidas nesse território. Muitas delas referem histórias de violências e processos de ruptura com familiares, inclusive com filhos, dos quais perderam a guarda, foram abrigados ou estão sob os cuidados de parentes.

Verifica-se também que parte das mulheres organiza suas rotinas com respostas imediatas às suas necessidades, que podem estar atreladas a carências e ao esforço de buscar meios de sobrevivência (para se alimentar, usar SPA, cuidar de terceiros e se proteger). Essas buscas, geralmente, colocam essas mulheres em situações de pouco ou nenhum poder, e por isso, com frequência, se expõem a relações abusivas. A questão da SPA, conjuntamente, se apresenta como um parâmetro para pensar o cuidado e a relação dessas mulheres com a rua. Afinal, o uso de SPA, muitas vezes, é relatado como mecanismo de sobrevivência às ausências (afetivas, 
estruturais e financeiras) e violências, presentes nas relações interpessoais e institucionais

\section{Primeiras abordagens e costura do vínculo com Cássia}

As primeiras abordagens de Cássia se deram em uma rua nomeada "Rua Morta", rua não asfaltada, sem casas convencionais ou prédios, mas com habitações de madeira, papelão ou sucata, em cuja extensão encontra-se a Unidade Básica de Saúde (UBS); no entanto, as pessoas que ali residem ou circulam dificilmente acessam esse serviço. Vale ressaltar que, nesse ponto da rua, há uma importante circulação de pessoas, usuárias de SPA, as quais permanecem a maior parte do tempo nesse espaço.

Nesse cenário, foram inúmeras as tentativas de aproximação com Cássia, que, muitas vezes, se mostrou afetuosa com a equipe, porém resistente às ofertas, consultas de pré-natal ou outras. A via de acesso, geralmente, eram os insumos da RD, água, piteira, protetor labial, soro fisiológico, produtos de higiene pessoal e o vínculo que tinha com os profissionais. Entretanto, conforme avançava sua gestação, foi possível realizar alguns atendimentos para avaliação dela e do bebê, na rua, na van e/ou na UBS próxima. Essas ações foram organizadas considerando o padrão de uso de SPA de Cássia, pois, por vezes, ou ela não estava em condições, ou retardava o início da ação ou, ainda, a interrompia por conta da dificuldade de permanecer sem usar.

Durante essas intervenções, Cássia afirmava que gostaria de ficar com 
o filho, que tinha outros dois filhos e que estavam bem, cuidados pela família extensa. Em um dos atendimentos, a usuária expôs sua dificuldade de pensar no parto e pós-parto, e afirmou desconhecer o recurso do abrigamento mãe-bebê. Sendo assim, foi realizada uma conversa sobre a possibilidade de inserção em um abrigo, o modo de organização da casa, os quartos, os móveis, a liberdade para decorar o ambiente conforme suas preferências e as atividades que ela poderia desenvolver, como participar de oficinas, da administração da rotina da casa e ter o apoio de uma equipe antes e após o parto. Cássia, de início, demonstrou desconfiança da oferta, porém se permitiu conhecer

\section{Ampliação dos recursos de suporte $e$ apoio}

Foram várias as tentativas de acompanhamento de Cássia até o abrigo, mas, por conta da intensidade do uso de SPA, da rede de relações que estabelecera na rua ou mesmo do receio de perder a guarda do bebê, ela se colocava resistente à visita à instituição. Portanto, as equipes do CnaR realizaram várias buscas ativas até conseguir encaminhá-la ao dispositivo. O primeiro encontro com o abrigo foi acompanhado de uma sensação de familiaridade, pois
Cássia conhecia muitas das mulheres assistidas.

Já a inserção de fato no serviço se deu por volta dos sete meses de gestação, e Cássia se adaptou ao espaço e às pessoas que lá circulavam de forma rápida. Logo teve seu quarto e ganhou roupas, bolsas e utensílios para uso pessoal e do bebê. As equipes do CnaR fizeram visitas, nas quais observaram a construção de desejos e expectativas relacionadas ao nascimento do bebê, pois a usuária pedia 
à equipe para tirar fotos para um álbum, mostrava seu quarto e demonstrava como gostaria que ficasse decorado; mostrava o espaço onde lavava as roupas; a cozinha onde preparava as refeições; e falava sobre sua relação com as outras mulheres e profissionais. Cássia também trazia à tona a dificuldade de manter contato com sua rede de relações e de manter-se na instituição sem o uso de SPA ou de outros recursos, como o doce, para lidar com a abstinência.

A questão do uso de SPA foi discutida com a usuária e entre as equipes do CnaR e do abrigo. Houve um espaço de troca entre os profissionais para refletir sobre o uso de SPA e a gestação, a ingesta de açúcar na gestação e amamentação, o uso de medicamentos para abstinência nesse período, dentre outras questões. A ideia não foi construir protocolos, mas compreender que o uso de SPA fazia parte da rotina e ocupava um lugar na organização daquelas mulheres, por isso deveria ser alvo de atenção das equipes e não de restrição/punição.

Apesar das tentativas de alinhamento, foi possível observar dificuldades com relação à criação de projetos singulares às moradoras. Por estarem inseridas em uma instituição, onde regras são colocadas para organização do trabalho, a oferta do açúcar, por exemplo, merece atenção, visto que, por conta da fissura, sentem necessidade de ingerir açúcar. Como consequência, algumas das mulheres abrigadas desenvolveram diabetes gestacional, quadro que exige uma dieta restritiva. A ponderação entre a oferta e restrição de açúcar exige disponibilidade dos profissionais para compreender as diferentes necessidades, que não estão descritas em uma cartilha, mas compõem o trabalho e refletem diretamente na vinculação e adesão das usuárias às propostas de abrigamento. 


\section{Sustentação de um modo de viver e implicação da instituição}

A permanência de Cássia na instituição foi permeada por muitas discussões quanto às suas necessidades, demandas e limites, próprios e da instituição. Foi interessante observar os arranjos e recursos da usuária, buscondo adaptarse às propostas com relação aos modos de "viver", tais como: a realização de atividades de autocuidado, de organização da casa, de participação em atividades de expressão e geração de renda, de uma rotina de cuidado com a saúde (acompanhamento na Atenção Básica e no Centro de Atenção Psicossocial álcool e drogas - CAPS AD), dentre outras. A adesão a esses recursos, e consequentemente a adaptação, não foi linear; Cássia saiu por vezes do abrigo, pernoitou fora, fez uso de SPA, mas sustentou sua permanência no serviço nos últimos meses da gestação.
Tais arranjos revelaram a implicação da usuária no projeto de vida movido pela gestação, assim como apontaram a necessidade de assistência a essa vivência como um direito. Nesse sentido, houve ensaios e ações diversas, no acompanhamento da Cássia, que não se restringiram somente às necessidades de saúde do bebê, mas no cuidado ampliado, abrangendo assistência, lazer, convivência e experiências.

A título de exemplo da amplitude e variedade das ações de cuidado das instituições, se faz necessária a descrição de um encontro do CnaR com a usuária no CAPS AD. Cássia estava participando da oficina de culinária, quando se direcionou aos profissionais, contando sobre seu envolvimento na organização de uma festa do abrigo, na qual haveria churrasco, piscina e comidas. Ela estava empolgada com a proposta e referiu 
MULHERES EM SITUAÇÃO DE RUA: UMA PERSPECTIVA DE CUIDADO SINGULARIZADO, TERRITORIAL E INTERSETORIAL

estar envolvida nas etapas da festa, como compra, arrecadação de dinheiro, dentre outras - demonstrando a diversidade das ofertas que envolvem a assistência a essa população, não restritas ao enfrentamento da SPA ou às condições clínicas e sociais.

Afinal, o envolvimento de Cássia na organização da festa está relacionado à ampliação do repertório de experiências, das possibilidades de se relacionar com o lazer, para alguém que, com frequência, se relaciona com o prazer de modo imediato, através da SPA, por exemplo. Além disso, a proposta envolve uma qualificação do tempo vivido na instituição.

\section{Diálogo com a experiência de gestação da mulher em situação de rua}

\section{A gestação de cuidado da mulher}

A descrição dos encontros com as mulheres e, em especial, com uma gestante em situação de rua, aponta para o fato de a maternidade não ser uma questão puramente biológica, mas um fenômeno social, com inscrição cultural, carregado de valores e marcas, tanto sociais quanto subjetivas. Estudos, como o de Belloc, Cabral e Oliveira (2018) e Gontijo e Medeiros (2008), assinalam que o período de gestação ou a intenção de ter um filho se coloca como fator mobilizador na construção de projetos de vida ou na transformação de cotidianos, principalmente, para mulher.

Assim, a gestação ou a maternidade para as mulheres em situação de rua são condições carregadas de significados, que extrapolam a concepção de família, pois dizem respeito a uma série de desejos de transformações ou não nos modos de produção de vida, ligados às 
MULHERES EM SITUAÇÃO DE RUA: UMA PERSPECTIVA DE CUIDADO SINGULARIZADO, TERRITORIAL E INTERSETORIAL

experiências pregressas e expectativas. Gontijo e Medeiros (2008), por exemplo, ao estudar a maternidade de adolescentes em situação de rua, registram dois significados atribuídos aos filhos por essas mulheres e destacam o potencial transformador de tais.

O primeiro remete o filho à condição daquele que irá acabar com a solidão, com o abandono vivenciado pelas adolescentes. Esse significado se sustenta pela crença de uma relação duradoura, sendo o filho considerado a única forma de dar e receber amor, de forma genuína [...]. O segundo significado atribuído ao filho foi a visão do filho como 'salvador' de uma morte certa nas ruas, representando a possibilidade de construção de um futuro, que para acontecer implica a saída da adolescente do espaço da rua. (Gontijo e Medeiros, 2008, p. 471).

Esses significados não são exclusivos das adolescentes nas ruas. No artigo produzido por Belloc, Cabral e Oliveira (2018), a questão da ampliação das possibilidades de vida mobilizadas pela gestação ou maternidade também são apresentadas, em detrimento dos recursos reais dos pais, de vida, moradia, suporte afetivo e financeiro. Da mesma forma, a gestação de Cássia se colocou como fator transformador, pois ela passou a morar em um abrigo, conviver com mulheres e crianças e acessar serviços de cuidado. Ou seja, sua rotina e a relação com os bens de consumo e lazer se transformaram, imprimindo assim um outro ritmo, um outro modo de estar e existir. A vida de Cássia adquiriu um jeito próprio, construído a partir de sua história, condições subjetivas, físicas e sua rede de suporte. Trata-se de um modo novo de produção de vida, conforme descrito por Quarentei (2001).

O receio de perder a guarda dos filhos não sem fundamento, haja vista a repetição do acolhimento dos filhos de mulheres em situação de rua (Belloc et al., 2018) - similarmente, é responsável pela produção de transformações no cotidiano e organização de vida dessas mulheres, que podem passar a procurar um lugar fixo de moradia, acessar serviços de assistência social e saúde, planejar e organizar meios para garantir a alimentação e o autocuidado, a médio 
MULHERES EM SITUAÇÃO DE RUA: UMA PERSPECTIVA DE CUIDADO SINGULARIZADO, TERRITORIAL E INTERSETORIAL

e longo prazo, entre outras transformações. De fato, as instituições e órgãos de proteção e assistência de mulheres e crianças possuem protocolos de trabalho embasados num modelo do "viver", da "família" e de relação "mãebebê", ou seja, exigem das mulheres disponibilidade e organização psíquica para exercício da maternidade, para enquadramento.

Além disso, a gestação ocasiona uma série de transformações no cotidiano e nas relações das mulheres, que requerem adaptações e podem revelar a necessidade ou não de acessar instituições, pois elas sofrem com alterações no sono, nas relações sexuais, na alimentação e até mesmo nas atividades miccionais (Costa et al, 2015). A gestação avançada também expõe a maternidade, o que pode mobilizar socialmente outras pessoas e ampliar as redes de apoio (Costa et al, 2015). Essas transformações e mobilizações se dão a partir da compreensão de um cotidiano com fronteiras e barreiras diluídas, sem demarcações sociais, no qual se dá a mobilidade, a desterritorialização, o nomadismo (Costa, 2005, citado por Ricci e Leal, 2016)

\section{Desvios, apropriações e legitimações de violências institucionais no processo do "viver"}

A gestação e/ou maternidade de mulheres em situação de rua mobiliza as equipes do SUS e SUAS, devido à complexidade, responsabilidade sociosanitária e necessidade de garantir a proteção e o acesso a direitos tanto à mulher quanto ao bebê. No entanto, alguns estudos sinalizam uma série de lacunas no cuidado dessas mulheres que, muitas vezes, se veem num ciclo entre rua, abrigamento, gravidez, plano de família e perda da guarda da criança; um ciclo que pode ser reforçado pelas ações intersetoriais (Belloc et al., 2018).

Nada obstante, a descontinuidade, a preocupação restrita à criança, a 
MULHERES EM SITUAÇÃO DE RUA: UMA PERSPECTIVA DE CUIDADO SINGULARIZADO, TERRITORIAL E INTERSETORIAL

necessidade de inserção das mulheres em uma instituição e os preconceitos reforçam a repetição da retirada e acolhimento institucional dos filhos. Belloc et al. (2018), ao se depararem com a reiteração desses fatos na vida das mulheres, supõem que isso não decorre de fracassados protocolos de cuidado, mas que os "[...] protocolos (oficiais ou informais) foram construídos na perspectiva do fracasso, ou seja, na suposição de que estas mães estavam fadadas a fracassar nas suas funções de maternidade" (Belloc et al., 2018, p. 43).

Belloc et al. (2018) evidenciam ainda que esses protocolos estão inscritos num processo de desmaternização, conduzido intersetorialmente como causa e efeito de um modelo excludente de sociedade, respaldado na ausência de políticas públicas. No caso registrado neste artigo, por exemplo, podem-se destacar os momentos de vida da usuária. Cássia se propõe a morar em um abrigo, tenta manter uma rotina de cuidado consigo e o bebê, tenta seguir regras e orientações das equipes, no entanto, observam-se lacunas entre o cuidado ofertado e suas reais necessidades. É o que se pode perceber na dificuldade de alinhamento entre as equipes do SUS e SUAS, pois há no país uma política norteadora das ações direcionadas a usuários de SPA que coloca a RD como estratégia, no entanto, suas práticas não são de conhecimento geral, impossibilitando assim a construção de um projeto compartilhado de assistência.

Cássia fazia uso abusivo de SPA, porém, quando institucionalizada, suspendeu, sem a oportunidade de adotar estratégias para lidar com as demandas da abstinência, como a ingestão de açúcar, o deslocamento até seu território de vida e relações, a substituição por uma substância menos danosa, entre outras, - que pode ter dificultado sua permanência no abrigo. Destaque-se que a RD não condiciona $a$ atenção à abstinência, mas sim à adoção de uma série de estratégias que podem viabilizar e ampliar o processo de escolha dos sujeitos (Surjus e Silva, 2019). Novamente, não é pretensão apontar falhas, mas atentar para a capacidade de legitimar e reforçar processos de tutela e violência. Todavia, as mulheres não podem ser penalizadas pelas carências 
MULHERES EM SITUAÇÃO DE RUA: UMA PERSPECTIVA DE CUIDADO SINGULARIZADO, TERRITORIAL E INTERSETORIAL

de recursos estruturais e políticos que consigam responder às suas reais necessidades. Nesse sentido, afirmam Macerata et al, 2014, p. 924:

Vemos modos de intervenções que, na sua grande maioria, tentam lidar com a rua de duas formas: a primeira é a de simples inclusão das pessoas em situação de rua, com o objetivo de moldar e adaptar seus modos de vida aos modos considerados normais, ou 'saudáveis'. O outro é de uma relação de isolamento ou extermínio.

\section{Novos cenários políticos}

Ao longo dos encontros, foi perceptível que parâmetros de sucesso e insucesso são difíceis de mensurar quando se trata de cuidado singular. No entanto, há ações construídas a partir das políticas públicas nos territórios dos sujeitos que apontam para uma potência de desvio, de ruptura com repetidos ciclos de violência e negligência; apontam para uma capacidade de ressignificar histórias, relações e transformar os modos de produção de vida em alvo de atenção.

No entanto, como já foi registrado, a história da saúde no Brasil reforça o processo de exclusão de pessoas em situação de rua. Foram anos de transformações políticas até construir o que compreendemos como RAS e RAPS. Porém, toda a potência libertadora e autonomizadora dessas redes estão à mercê de mudanças, visto os novos arranjos políticos, como a Norma Técnica n¹1/2019 (Brasil, 2019), que retoma o financiamento de hospitais psiquiátricos; não reconhece a proposta dos serviços substitutivos; prevê o financiamento de ambulatórios; propõe o combate às drogas e não a RD, dentre outras.

Ou seja, configuram propostas de caráter assistencialista, pouco inclusivas, como afirmam Costa et al.:"O caráter 
MULHERES EM SITUAÇÃO DE RUA: UMA PERSPECTIVA DE CUIDADO SINGULARIZADO, TERRITORIAL E INTERSETORIAL

assistencial pode inibir/interferir no desenvolvimento da autonomia desses sujeitos, uma vez que inibe as possibilidades de poder contratual [...]" (2015, p. 1095). Com isso, tendem a perpetuar um laço de dependência entre o usuário e a instituição, mantendo-o numa postura passiva e tutelada (Costa

\section{Considerações finais}

A experiência no CnaR exaltou a necessidade de publicizar o que se dá no cotidiano das equipes com a população; o que se dá na assistência direcionada à significação da cidadania, à ampliação da assistência e à organização em rede. Afinal, essa forma de cuidar propõe-se a romper com a invisibilidade, a acolher as diferentes formas de "viver" e, consequentemente, a superar o modelo assistencialista.

Além disso, o encontro com as mulheres em situação de rua e, em especial, com Cássia, escancarou a complexidade das et al, 2015), visto que as "[...] redes institucionais também alimentam esse circuito de rua, produzindo sua clientela e sendo produzido por ela [...]" (Frangella, 2004, p. 32). Portanto, há de se pensar a clínica, o trabalho e as instituições como movimentos políticos de defesa de um modelo de assistência.

situações vivenciadas, assim como a mobilização que a gestação provoca nas equipes de cuidado. No entanto, a saúde é um direito a priori dessas mulheres, não restrito a essas condições. Portanto, como contribuição desta escrita, em conjunto com os autores citados, fica a validação das práticas territoriais, integrais, intersetoriais e equânimes, que têm como objetivo a cidadania, o viver, os desejos, limites e condições dos usuários. Esta contribuição é, também, o desafio para o meio acadêmico, político e prático. 


\section{Referências}

Belloc, M.M., Cabral, K.V., \& Oliveira, C.S. (2018). A desmaternização das gestantes usuárias de drogas: violações de direitos e lacunas do cuidado. Saúde em Redes, 4 (1), pp. 37-49). DOI: hp://dx.doi.org/10.18310/24464813.2018v4n1suplemp37-49.

Carvalho, G. (2013). A Saúde Pública no Brasil. Estudos avançados, 27(78). São Paulo, SP.

Costa, S.L., Vida, C.P.C., Gama, I.A., Locatelli, N.T., Karam, B.J. Ping, C.T., \& Bernardes, A.F.M. (2015). Gestantes em situação de rua no município de Santos, SP: reflexões e desafios para as políticas públicas. Saúde e Sociedade, 24 (3), pp. 1089-1102). São Paulo, SP.

Frangella, S.M. (2004). Corpos urbanos errantes: uma etnografia da corporalidade de moradores de uma rua em São Paulo. Tese de doutorado, Instituto de Filosofia e Ciências Humanas, Universidade Estadual de Campinas). Recuperado de http://www.repositorio.unicamp.be/handle/REPOSIT/279907.

Gontijo, D.T., \& Medeiros, M. (2008). "Tava morta e Revivi" : significado de maternidade para adolescentes com experiência de vida nas ruas. Saúde Pública, 24 (2), pp. 469-472. 
MULHERES EM SITUAÇÃO DE RUA: UMA PERSPECTIVA DE CUIDADO SINGULARIZADO, TERRITORIAL E INTERSETORIAL

Jorge, C.F., \& Ricci, E.C. (2019). Mulheres em Situação de Rua: gestação, maternidade e cuidado intersetorial na perspectiva da produção de vida. Trabalho de Conclusão de Residência, Faculdade de Medicina, Unicamp).

Recuperado de https://www.fcm.unicamp.br/fcm/sites/default/files/2019/page/tcc_final_caroli ne.pdf.

Macerata, I., Soares, J.G.N., \& Ramos, J.F.C. (2004). Apoio como cuidado de territórios existenciais: atenção básica e a rua. Interface, (18)1, pp. 919-930.

Nery Filho, A., Valério, A.L.R., \& Monteiro, L.F. (2011). Guia do projeto consultório de rua. Brasília, DF: SENAD. Salvador, BA: CETAD.

Nota técnica $n^{\circ}$ 11/2019. (2019). Brasil. Ministério da Saúde. Secretaria de Atenção à Saúde. Departamento de Ações Programáticas Estratégicas. Coordenação-Geral de Saúde Mental, Álcool e Outras Drogas. Brasília, DF: Ministério da Saúde.

Política Nacional de Atenção Básica. (2012). Brasil. Ministério da Saúde. Secretaria de Atenção à Saúde. Departamento de Atenção Básica. Brasília, DF: Ministério da Saúde.

Política Nacional de Atenção Integral à Saúde da Mulher: princípios e diretrizes. (2004). Brasil. Ministério da Saúde. Secretaria de Atenção à Saúde. 
MULHERES EM SITUAÇÃO DE RUA: UMA PERSPECTIVA DE CUIDADO SINGULARIZADO, TERRITORIAL E INTERSETORIAL

Departamento de Ações Programáticas Estratégicas. Brasília, DF: Ministério da Saúde.

Política Nacional para Inclusão Social da População em Situação de Rua. (2008). Brasil. Governo Federal. Brasília, DF: Ministério da saúde.

Prudente, T.C.B., Gontijo, D.T., \& Paiva, R.B.C. (2018). Desempenho ocupacional de mulheres em situação de rua. Rev. Interinst. Bras. Ter. Ocup. (2)1, pp. 85-108.

Quarentei, M.S. (2001). Conferência de Encerramento: Terapia Ocupacional e Produção de Vida" . VII Congresso Brasileiro de Terapia Ocupacional. Porto Alegre, RS.

Ricci, E.C., \& Leal, EM. (2016). Cotidiano, esquizofrenia e narrativas da experiência de adoecimento. São Carlos, SP: Cadernos Brasileiros de Terapia Ocupacional.

Surjus, L.T.L.S., \& Silva, P.C. (2019). Redução de Danos: Ampliação da vida e materialização de direitos. Santos, SP. Recuperado de https://www.unifesp.br/campus/san7/images/E-book-Reducao-Danos-versao$\underline{\text { final.pdf }}$

Recebido em: 21/08/2020

Aprovado em: $15 / 12 / 2020$ 